Marquette University

e-Publications@Marquette

$1-1-1998$

\title{
Photoinduced Coupling of Acetylenes and Quinone in the Solid State as Preorganized Donor-Acceptor Pairs
}

E. Bosch

University of Houston

S. M. Hubig

University of Houston

Sergey V. Lindeman

Marquette University, sergey.lindeman@marquette.edu

Jay K. Kochi

University of Houston

Accepted version. The Journal of Organic Chemistry, Vol. 63, No. 3 (1998): 592-601. DOI. (C) 1998 American Chemical Society. Used with permission.

Sergey V. Lindeman was affiliated with the University of Houston at the time of publication. 
Marquette University

e-Publications@Marquette

\title{
Chemistry Faculty Research and Publications/College of Arts and Sciences
}

This paper is NOT THE PUBLISHED VERSION; but the author's final, peer-reviewed manuscript. The published version may be accessed by following the link in the citation below.

Journal of Organic Chemistry, Vol. 63, No. 3 (January 22, 2008): 592-601. DOI. This article is (C) American Chemical Society and permission has been granted for this version to appear in ePublications@Marquette. American Chemical Society does not grant permission for this article to be further copied/distributed or hosted elsewhere without the express permission from American Chemical Society.

\section{Photoinduced Coupling of Acetylenes and Quinone in the Solid State as Preorganized Donor-Acceptor Pairs}

\section{E. Bosch}

Chemistry Department, University of Houston, Houston TX

S. M. Hubig

Chemistry Department, University of Houston, Houston TX

S. V. Lindeman

Chemistry Department, University of Houston, Houston TX

J. K. Kochi

Chemistry Department, University of Houston, Houston TX

\begin{abstract}
Crystalline electron donor-acceptor (EDA) complexes of various diarylacetylenes (DA) and dichlorobenzoquinone (DB) are isolated and structurally characterized by X-ray crystallography.
\end{abstract}


Deliberate excitation of either the DB acceptor at $\lambda_{\mathrm{DB}}=355 \mathrm{~nm}$ or the $1: 2$ [DA, 2DB] complex at $\lambda_{\mathrm{CT}}=$ $532 \mathrm{~nm}$ in the solid state leads to [2+2] cycloaddition and identical (isomeric) mixtures of the quinone methide products. Time-resolved (ps) diffuse reflectance spectroscopy identifies the ion-radical pair $\left[\mathbf{D A}^{\bullet+}, \mathbf{D B}^{\cdot-}\right]$ as the reactive intermediate derived by photoinduced electron transfer in both photochemical procedures. The effects of crystal-lattice control on the subsequent ion-radical pair dynamics are discussed in comparison with the same photocouplings of acetylenes and quinone previously carried out in solution.

\section{Introduction}

Stereospecific [2 + 2] cycloadditions of crystalline olefinic derivatives (dimerization) can be achieved by solid-state photochemistry. $\underline{1}$ Thus, elegant studies by Schmidt and co-workers 2,3 have shown the formation of $\alpha$-truxillic and $\beta$-truxinic acids to proceed via the $\alpha$ - and $\beta$-crystalline modifications in which the cinnamic acid molecules are stacked in head-to-tail and head-to-head conformations, respectively. Importantly, the $\gamma$-modification in which the cinnamic acid is also packed head-to-head, but the molecules are staggered with respect to each other, is unreactive, i.e.

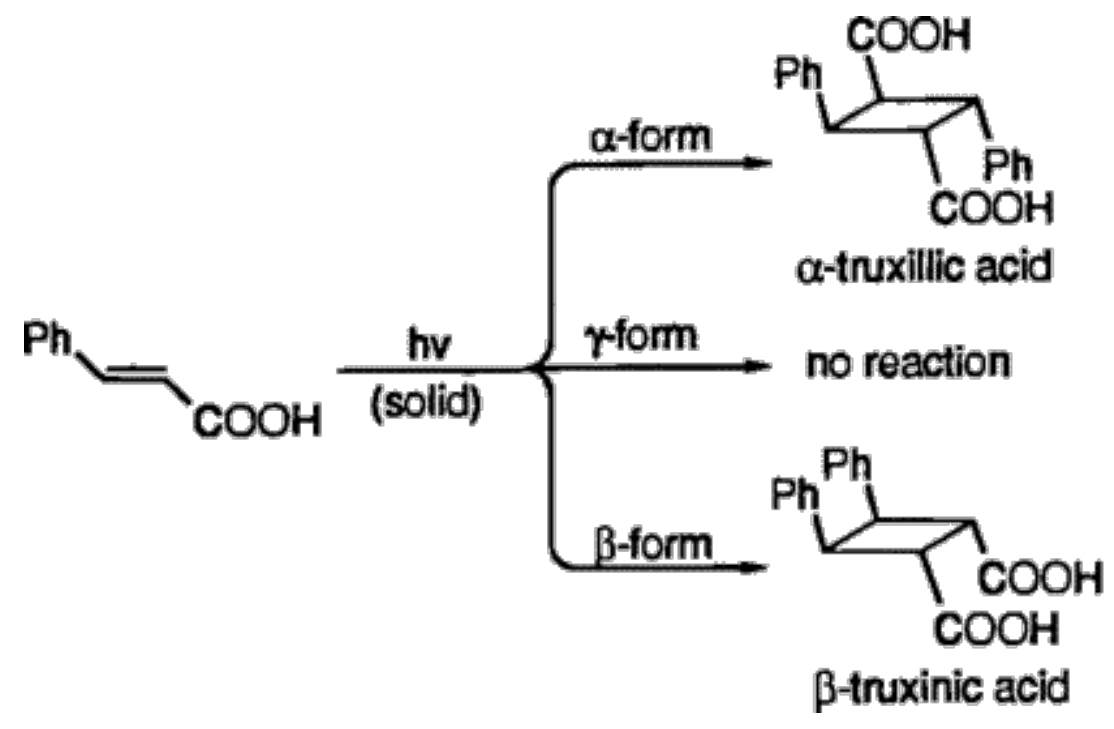

Such a clear display of lattice control over reactivity and stereochemistry was correlated with a wealth of crystallographic data on related systems, and it resulted in the formulation of the topochemical postulate that states: 2,4 "reaction in the solid state must take place such that there is the least movement of the atoms within the crystal lattice". In the particular case of cycloadditions of olefins, it was empirically determined that the olefins should ideally lie in parallel planes with the double bonds oriented in the same direction with a maximal distance between the olefins of $4.2 \AA{ }^{5}$

Subsequent studies on solid-state [2 + 2] cycloadditions have been largely limited to olefin dimerizations $\frac{7,8}{2}-$ most notably a systematic study of the dimerization of a series of coumarin derivatives, $\underline{9}$ all of which obeyed Schmidt's topochemical postulate. ${ }^{2}$ The major problem in extending Schmidt's postulate to other [2 +2] cycloadditions lies in the difficulty in forming crystalline lattices that contain unlike molecules such that the reactive centers have the correct alignment for reaction. Indeed, several isolated reports of solid-state [2 +2$]$ cycloadditions of dissimilar molecules have 
generally involved molecules with only minor modifications between them, $\underline{10,11}$ e.g. in a mixed crystal where $\mathrm{Ar}=p-\mathrm{ClC}_{6} \mathrm{H}_{4}$ and $\mathrm{Ar}^{\prime}=p-\mathrm{MeOC}_{6} \mathrm{H}_{4}$.

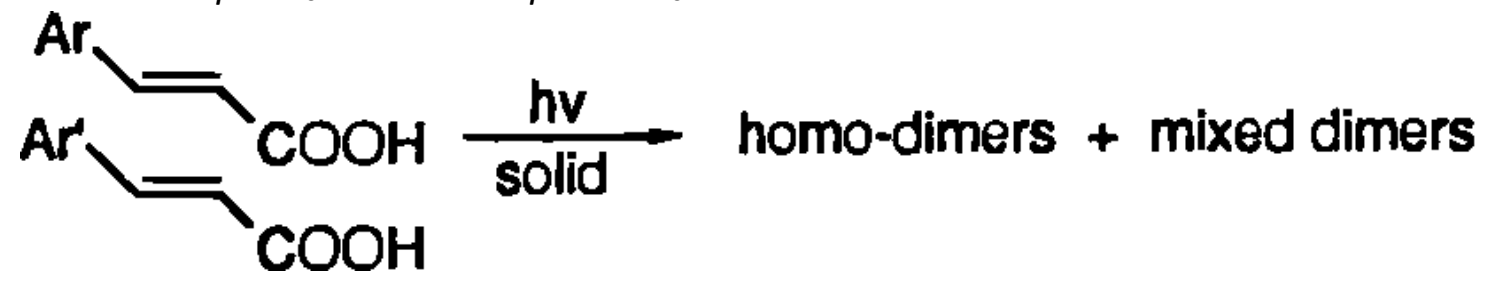

We now report on a new approach to solid-state [2 + 2] cycloadditions, in which the preequilibrium formation of weakly bound electron donor-acceptor (EDA) complexes is utilized to engineer crystals in which a pair of dissimilar (reactive) partners can attain an orientation suitable for [2 + 2] photocoupling. In this regard, we note that benzoquinones form a wide range of highly colored EDA

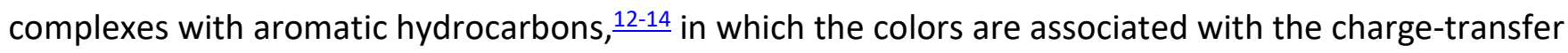
(CT) absorption band of the donor-acceptor interaction. $\frac{15}{A}$ As a result, such EDA complexes are subject to photoactivation by either (a) the direct irradiation of the acceptor or (b) the specific excitation of the CT absorption band of the EDA complex. $\underline{16}$ For example, we recently showed that the [2+2] cycloaddition of various diarylacetylenes (DA) with 2,6-dichlorobenzoquinone (DB), i.e.

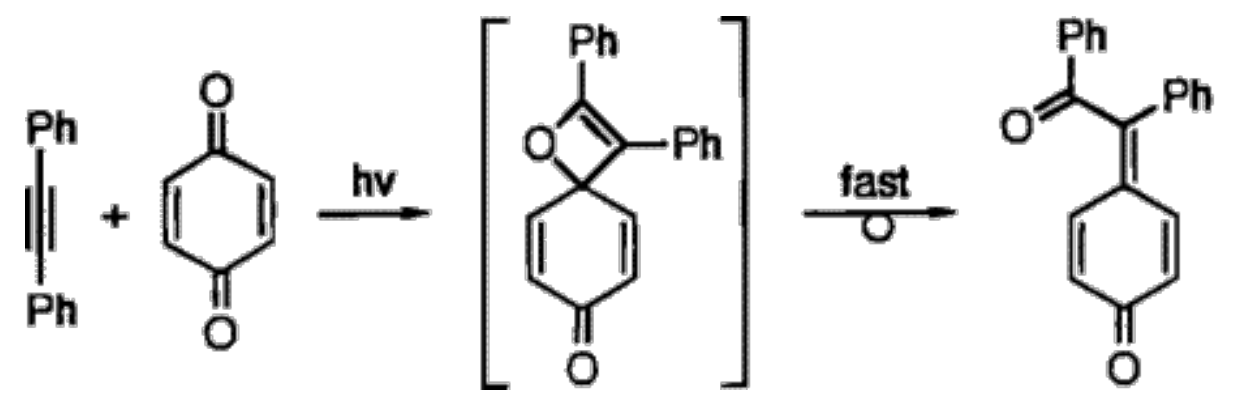

can be readily effected to high yields in solution by direct photoexcitation of the acceptor DB with actinic irradiation at $\lambda_{\text {exc }}=355 \mathrm{~nm}$ or by specific photoactivation of the 1:1 EDA complex [DA,DB] at $\lambda_{\text {exc }}$ $=532 \mathrm{~nm} \cdot \frac{17,18}{}$ Since the parent compounds in eq 3 ( $p$-benzoquinone and tolane) showed limited charge-transfer activity, we prepared a series of the polymethyl derivatives in Chart 1 to afford with 2,6-dichlorobenzoquinone (DB) a series of brightly colored complexes. Thus, we anticipated this group of acetylene donors to provide a suitable range of related crystalline environments to study the solidstate photocoupling in crystalline EDA complexes.

The acronyms reflect the pattern of methyl substitution on each of the phenyl groups, i.e., $\mathbf{F}=$ phenyl, $\mathbf{T}=$ tolyl, $\mathbf{X}=m$-xylyl, $\mathbf{X}^{\prime}=p$-xylyl, $\mathbf{H}=$ hemimellityl, $\mathbf{M}=$ mesityl, and $\mathbf{P}=$ pentamethylphenyl with $0,1,2$, 3,4 , and 5 methyl substituents, respectively.

\section{Results}

\section{Preparation of Crystalline EDA Complexes of Diarylacetylene Donors with 2,6-}

Dichlorobenzoquinone. Immediately upon the mixing of equimolar amounts of the colorless crystalline tolyl- $m$-xylylacetylene donor TX with canary yellow crystals of acceptor DB, the solid mixture took on an orange coloration. The solid was dissolved in the minimum amount of dichloromethane and the 
solvent allowed to slowly evaporate at $-20^{\circ} \mathrm{C}$ in the dark. As the dichloromethane evaporated, orange needles precipitated from the solution together with colorless crystals of unchanged TX. Quantitative NMR and GC analysis of the orange needles established a 2:1 stoichiometry of DB and TX. Several additional experiments confirmed that the same 2:1 molar ratio of acceptor to donor was obtained, irrespective of the initial amounts of TX and DB present in the solution (ranging from 1:2 to 4:1), i.e.

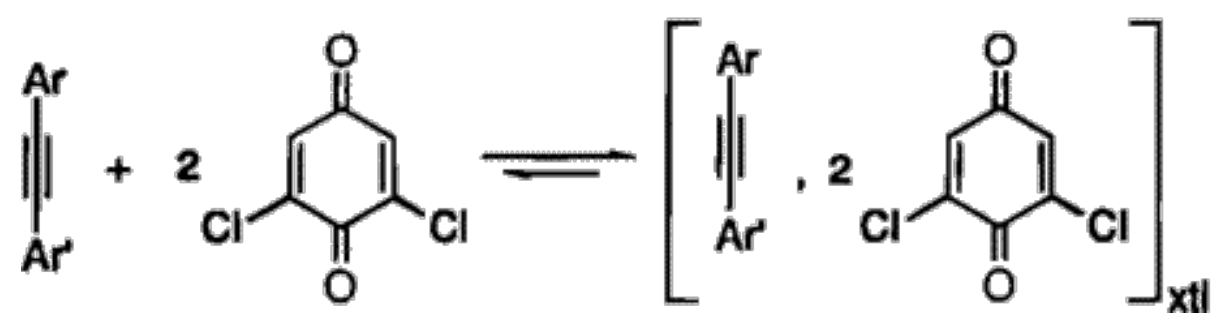

where $\mathrm{Ar}=p$-tolyl and $A r^{\prime}=m$-xylyl. Similarly colored crystals were also grown from dichloromethane solution of dichlorobenzoquinone DB with the other diarylacetylenes $\mathbf{X X}, \mathbf{T M}, \mathbf{X M}, \mathbf{X H}$, and FP. Analysis of these complexes confirmed that they were all comprised of 2:1 mixtures of DB and the acetylene donor. The weakest donor in the series, phenyl-m-xylylacetylene FX, did not show any change in coloration on mixing in the solid state with DB. However, this colorless mixture on dissolution in dichloromethane formed a pale orange solution that upon evaporation deposited a microcrystalline yellow/orange solid. By contrast, immediately upon mixing phenyl(mesityl)acetylene FM with DB, the solids melted together to form a red oil, but crystals were not formed.

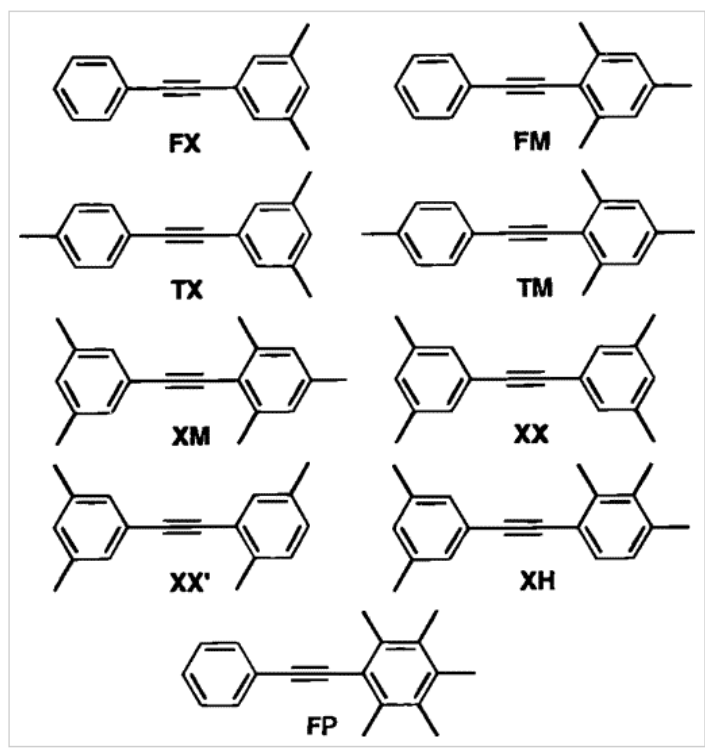

Chart 1

II. Spectral (UV-Vis) Characterization of Crystalline Electron Donor-Acceptor Complexes. The 2:1 crystalline solids formed between DB and the graded series of diarylacetylene donors in Chart 1 were 
characterized by a progressive reddening that was correlated with the extent of methyl substitution. Thus, mixtures of dichlorobenzoquinone with tolyl-m-xylylacetylene (TX) yielded pale orange crystals, di- $m$-xylylacetylene $(\mathbf{X X})$ yielded orange crystals, and dark red crystals were formed from phenyl(pentamethylphenyl)acetylene (FP). To quantify these visual effects, we recorded the UV-vis diffuse-reflectance spectra of the EDA crystals. For example, a solid mixture of the yellow/orange crystalline complex [TX,2DB] diluted in silica (1 wt \%) was ground until a homogeneous mixture was obtained. The pale orange solid was transferred to a 1-mm quartz cuvette and the diffuse reflectance spectrum recorded. Separately, the diffuse reflectance spectrum of pure DB was recorded as a mixture in silica such that the concentration of DB matched that in the colored complex. Spectral examination (Figure $1 \mathrm{~A}$ ) revealed that the absorption band of DB at $350 \mathrm{~nm}$ had approximately the same intensity in both spectra. Digital subtraction of the latter spectrum from the former generated the broad absorption (Gaussian) spectrum with the maximum at $\lambda_{C T}=500 \mathrm{~nm}$ shown in Figure $1 \mathrm{~B}$. The absorption maxima of the other crystalline EDA complexes obtained by a similar procedure are listed in Table 1.

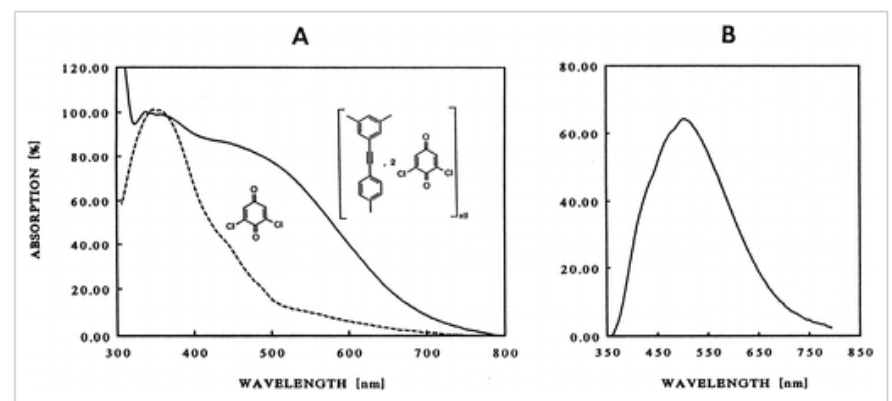

Figure 1 (A) Diffuse reflectance spectrum of the crystalline EDA complex of tolyl- $m$-xylylacetylene (TX) with dichlorobenzoquinone (DB) recorded as a (1 wt \%) dispersion in silica. The dashed curve represents the diffuse reflectance spectrum of dichlorobenzoquinone alone under the same conditions (see text). (B) Charge-transfer band of the TX,2DB complex obtained by digital subtraction of the spectra in A.

Table 1. Diffuse Reflectance Spectra of Crystalline (2:1) EDA Complexes of 2,6-Dichlorobenzoquinone with Diarylacetylene Donors

$\begin{array}{lllll}\text { DA donor } & E_{\mathrm{ox}}^{b}(\mathrm{~V} \text { vs SCE }) & \text { DB acceptor } \lambda_{\mathrm{DB}}^{c, d}(\mathrm{~nm}) & \lambda_{\mathrm{CT}}^{c, e}(\mathrm{~nm}) & \mathrm{fwhm} \\ \text { TX } & 1.61 & 350 & 500 & 99 \\ \mathbf{X X} & 1.57 & 350 & 496 & 101 \\ \mathbf{X M} & 1.48 & 350 & 524 & 91 \\ \mathbf{X H} & 1.46 & 350 & 516 & 97 \\ \text { FP } & 1.46 & 350 & 520 & 96\end{array}$

${ }^{a}$ Identified in Chart $1 .{ }^{b}$ Peak potential of the irreversible anodic wave by $\mathrm{CV}$ at $\mathrm{v}=100 \mathrm{mV} \mathrm{s} \mathrm{s}^{-1}$ in dichloromethane solution containing $0.2 \mathrm{M} \mathrm{Bu}_{4} \mathrm{~N}^{+} \mathrm{PF}_{6}{ }^{-}$electrolyte. ${ }^{c}$ Diluted in silica (1 wt \%), see text and Experimental Section. ${ }^{d}$ Local band in the EDA complex (see Figure 1). ${ }^{e}$ Charge-transfer band in the EDA complex (see Figure 1). ${ }^{f}$ Full width at half-maximum. 
The relative donor strengths of the polymethylacetylene donors in Chart 1 were evaluated by a comparison of their oxidation potentials in solution, and the values of $E_{\text {ox }}$ are listed in Table 1 (arranged in order of increasing methyl substitution). The progressive red shift of the charge-transfer absorption band that is directly correlated with the donor strength of the acetylenes establishes the chargetransfer transition in the EDA complex, as formulated by Mulliken. ${ }^{19}$

III. Photoinitiated Coupling of Diarylacetylenes with 2,6-Dichlorobenzoquinone in the Solid State. The colored crystalline EDA complexes of the acetylene donors with DB (that were indefinitely stable in the dark) underwent a gradual bleaching when exposed to roomlight for extended periods. A systematic photochemical study was carried out as follows.

A. Direct Excitation. The deliberate photolysis of the crystalline EDA complex from di- $m$-xylylacetylene $\mathbf{X X}$ was carried out under controlled conditions by placing several crystals in a thin Pyrex tube that was sealed under an argon atmosphere. The reaction tube was placed in a clear Dewar filled with acetone cooled to $-60^{\circ} \mathrm{C}$ and the sample irradiated with a medium-pressure mercury lamp fitted with a sharp cutoff filter such that the sample was exposed only to visible light with $\lambda_{\text {exc }}>410 \mathrm{~nm}$. [The photolysis was performed at $-60^{\circ} \mathrm{C}$ in order to prevent local heating and melting of the EDA complex upon irradiation; see Experimental Section]. The tube was periodically rotated, and after $5 \mathrm{~h}$ the crystals were examined under a microscope. The crystals were extensively cracked and visibly darkened. They were dissolved in dichloromethane, and immediate analysis by quantitative GC indicated a 15\% conversion of the acetylene donor to a single quinone methide $\mathbf{2}$. The crude product was purified by thin-layer chromatography and found to consist of a 1:1 adduct, the quinone methide structure of which (see Figure 2 ) was established by X-ray structural analysis of a single crystal, ${ }^{20}$ i.e.

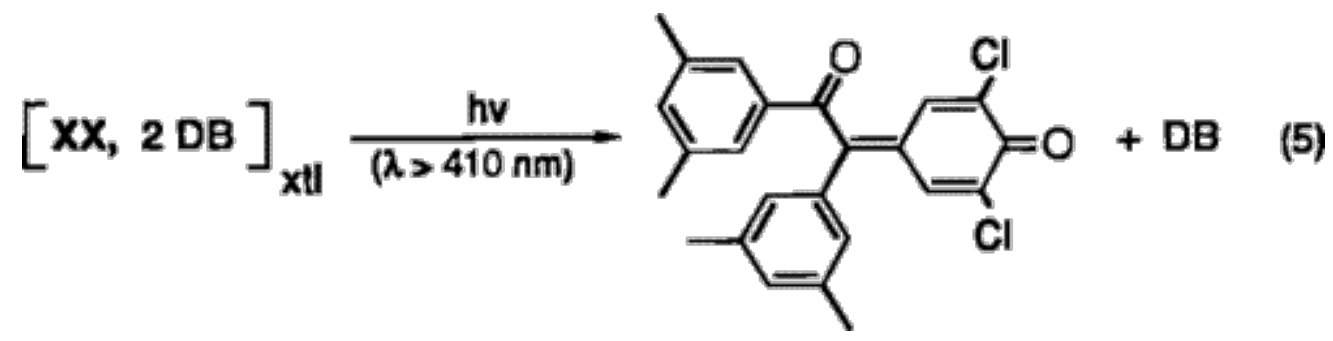

The EDA complexes of the acetylene donors with DB in Table 2 were similarly irradiated with $\lambda_{\text {exc }}>410$ $\mathrm{nm}$ at $-60^{\circ} \mathrm{C}$ to the various conversions (and yields) reported therein. The unsymmetrical diarylacetylenes generated a pair of isomeric products. For example, the photolysis of an equimolar solution of tolyl- $m$-xylylacetylene TX and DB yielded approximately a 4:1 mixture of two products. The regiochemistry of addition to the acetylene was readily established by analysis of the mass-spectral fragmentation pattern. In particular, the most abundant ion formed from the major isomer corresponded to the fragment $\mathrm{CH}_{3} \mathrm{C}_{6} \mathrm{H}_{4} \mathrm{CO}$ formed by preferential cleavage of the $p$-methylbenzoyl group. $\frac{21}{}$ On the other hand, the most abundant fragment in the minor product corresponded to the dimethyl analogue $\left(\mathrm{CH}_{3}\right)_{2} \mathrm{C}_{6} \mathrm{H}_{3} \mathrm{CO}$ and thereby established the methyl substitution pattern in the products. Since the proton chemical shifts and coupling of the vinylic protons in the products were similar to those recorded for the product from di-m-xylylacetylene in eq 5 , we concluded that the regiochemistry for the addition of the unsymmetrical dichloroquinone moiety was the same, i.e. 


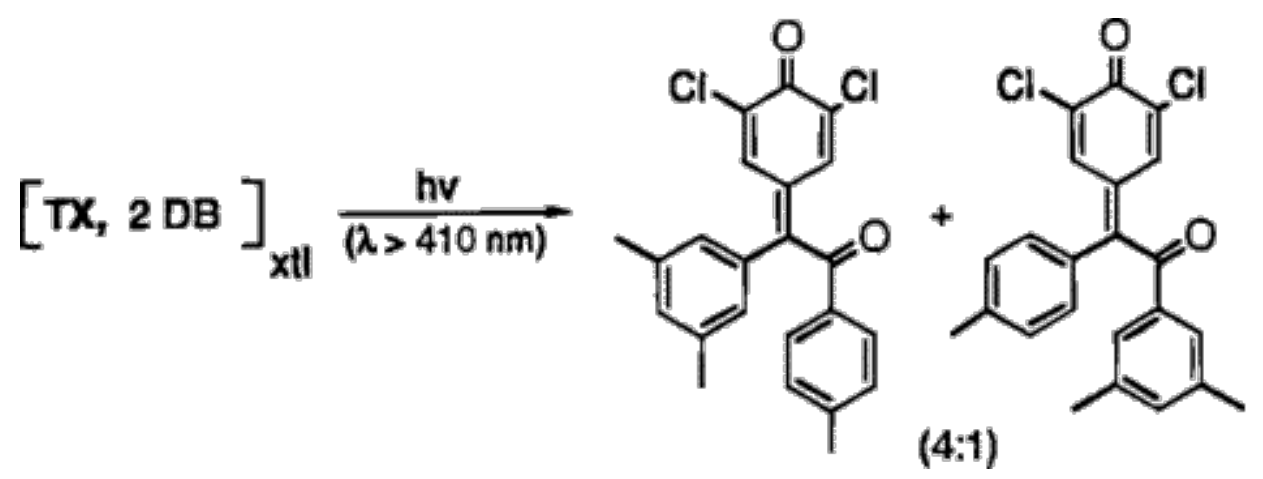

(6)

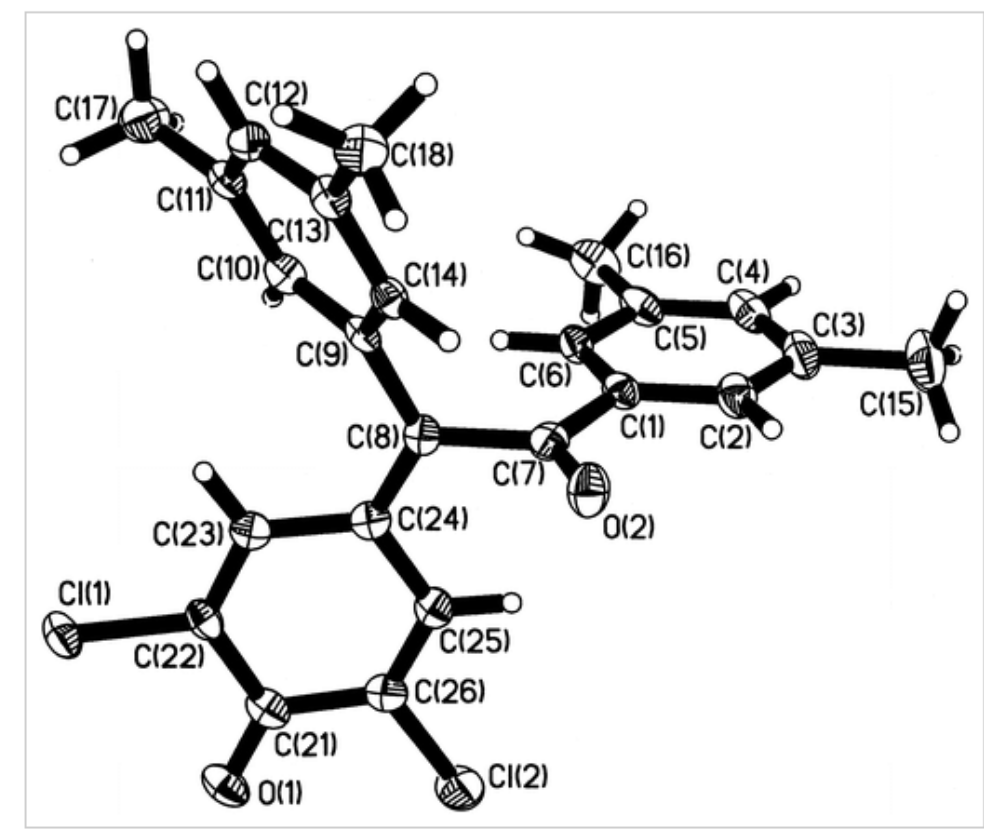

Figure 2 Molecular perspective of the 1:1 adduct obtained from the solid-state photoactivation of $\mathbf{X X}, 2 \mathrm{DB}$ to establish the quinone methide structure $\mathbf{2}$. 
Table 2. Photoinduced Coupling of the Crystalline EDA Complexes of Diarylacetylenes and 2,6Dichloroquinone ${ }^{a}$

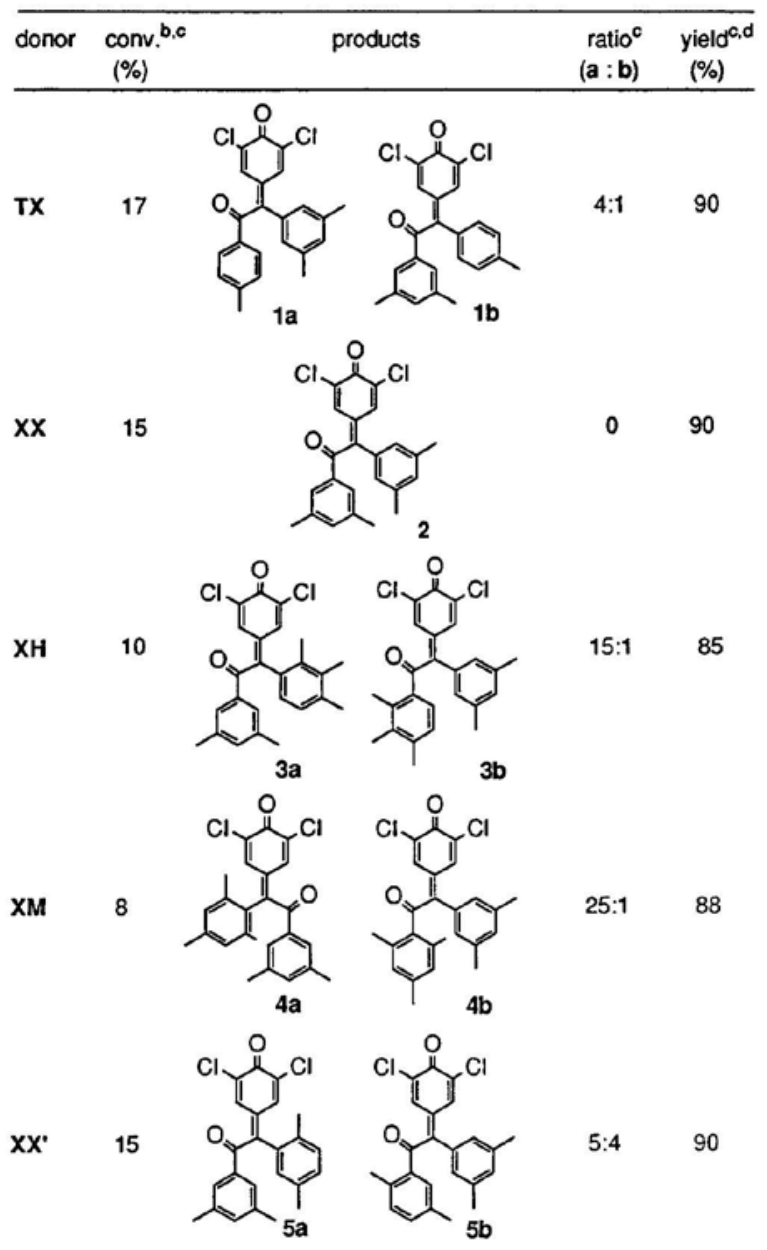

${ }^{a}$ Crystalline EDA complexes irradiated at $-60^{\circ} \mathrm{C}$ with a medium-pressure $\mathrm{Hg}$ lamp with a 410 -nm cutoff filter for $5 \mathrm{~h}$ (see Experimental Section). ${ }^{b}$ Based on donor. ${ }^{c}$ Determined by quantitative GC using an internal standard. ${ }^{d}$ Based on reacted donor. ${ }^{e}$ These crystals did not react on irradiation at $25^{\circ} \mathrm{C}$ for $24 \mathrm{~h} ; \mathbf{F P}$ and DB recovered in $>95 \%$ yield, traces $(<5 \%)$ of 2,6-dichlorohydroquinone detected by GC-MS.

The regiochemistry of addition in the other products in Table 2 was determined in a similar manner. The sole exception was the tetramethyl derivative $\mathbf{X} \mathbf{X}^{\prime}$, in which the isomeric products were not readily distinguished. Fortunately, the minor isomer readily crystallized from acetonitrile, and its structure was established by X-ray crystallography of a single yellow crystal ${ }^{20}$ (see Figure 3 ). It is noteworthy that addition to unsymmetrical acetylenes (except $\mathbf{X} \mathbf{X}^{\prime}$ ) generally proceeded with remarkable regioselectivity to favor the formation of the most substituted benzylidene and the least substituted benzoyl group as generically presented in eq 7 where $M$ represents the most substituted phenyl group and $L$ the least substituted group. 
<smiles>[M]C#CI</smiles>

major<smiles>[M]C(=O)C(I)=C1C=C(Cl)C(=O)C(Cl)=C1</smiles>

minor

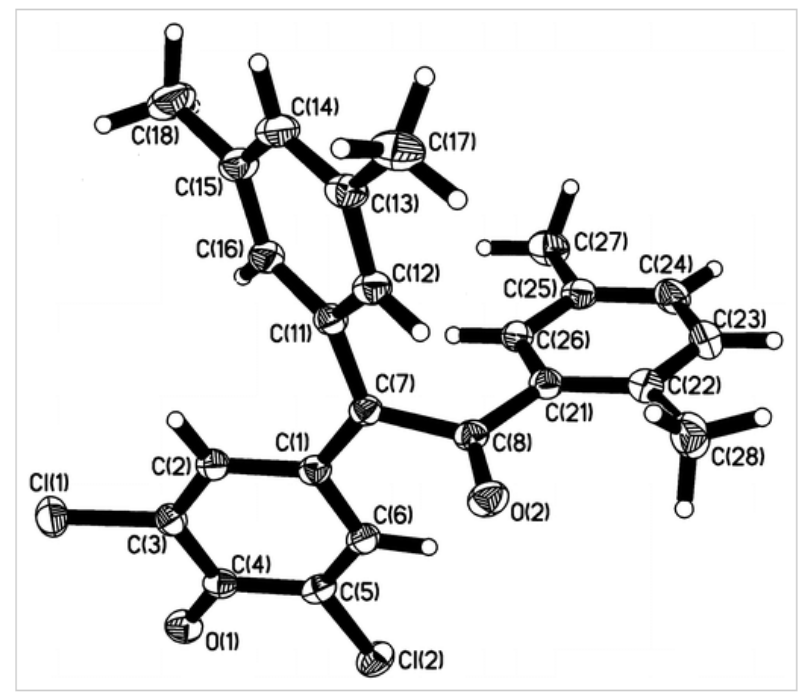

Figure 3 X-ray crystal structure of the minor regioisomer obtained by the photoactivation of $\mathbf{X X} \mathbf{X}^{\prime}, 2 \mathbf{D B}$ in the solid state, showing the different dispositions of the $m$-xylyl and $p$-xylyl groups in the quinone methide $\mathbf{5 b}$.

It was singularly noteworthy, however, that the EDA crystals of the phenyl(pentamethylphenyl)acetylene complex [FP,2DB] were unchanged after irradiation at $-60^{\circ} \mathrm{C}$ for $5 \mathrm{~h}$. Even when these crystals were irradiated with filtered light $(\lambda>410 \mathrm{~nm})$ at $25^{\circ} \mathrm{C}$ for prolonged periods of $>72 \mathrm{~h}$, the crystals visually appeared unchanged, and quantitative GC and NMR analysis confirmed that both FP and DB could be recovered intact.

B. Charge-Transfer Excitation. The broad charge-transfer absorption bands (see Figure 1) associated with the DB complexes with acetylene donors permitted the specific excitation of the EDA complex by performing the photolysis with light filtered through a sharp cutoff filter $\left(\lambda_{\text {exc }}>530 \mathrm{~nm}\right)$. Accordingly, the orange 2:1 crystals of tolyl-m-xylylacetylene TX with DB were sealed under an argon atmosphere in a thin Pyrex tube, which was placed in a clear Dewar filled with acetone cooled to $-60^{\circ} \mathrm{C}$. The crystals were irradiated with visible light from a $500-\mathrm{W}$ mercury lamp fitted with a cutoff filter with $\lambda_{\text {exc }}>530$ $\mathrm{nm}$ for $12 \mathrm{~h}$. Quantitative GC analysis indicated that a pair of isomeric quinone methides were formed $(\sim 5 \%)$ in a ratio of $>4: 1$, i.e. 


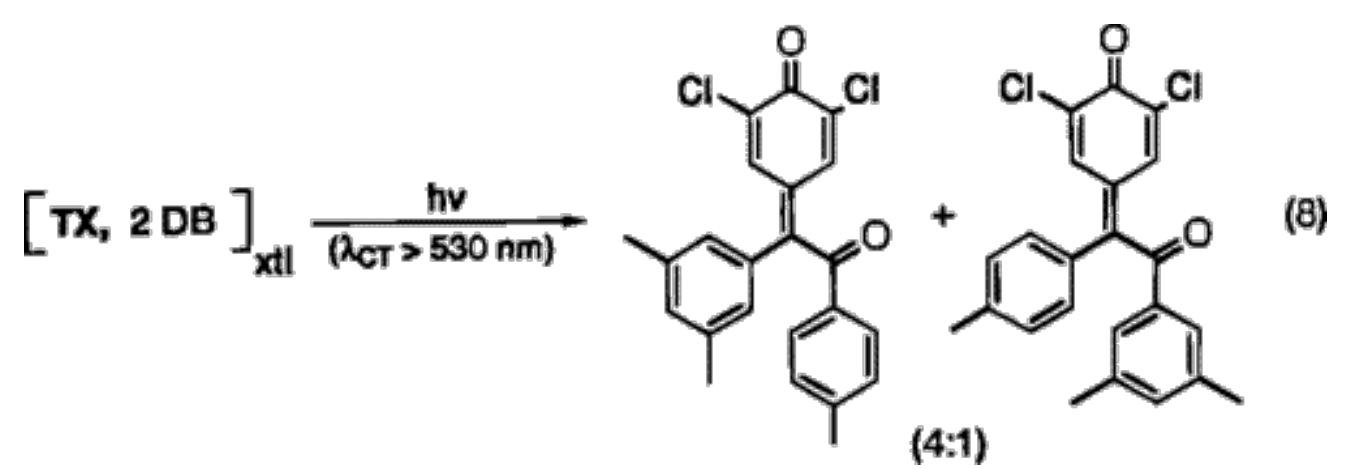

The EDA complexes of DB with the dixylylacetylenes $\mathbf{X X}$ and $\mathbf{X X}$ ' were also subjected to charge-transfer excitation, and they were similarly transformed to the quinone methides in Table 3 in the conversions and yields listed.

Table 3. Charge-Transfer Coupling of the Crystalline EDA Complexes of Diarylacetylenes and 2,6Dichloroquinone ${ }^{a}$

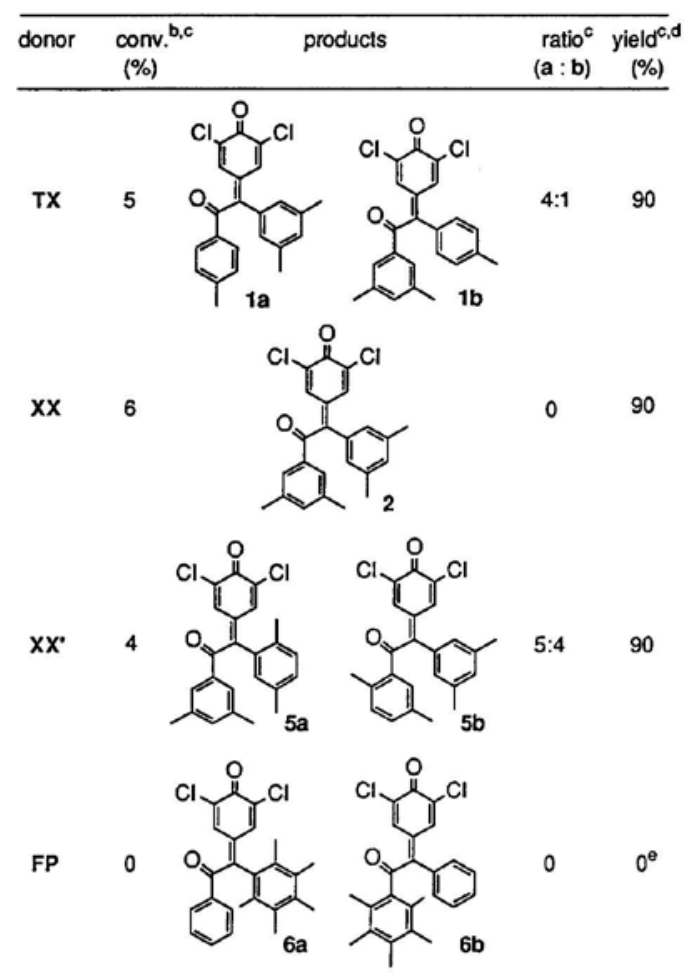

${ }^{a}$ Crystalline EDA complexes irradiated at $-60^{\circ} \mathrm{C}$ with a medium-pressure $\mathrm{Hg}$ lamp with a $530 \mathrm{~nm}$ cutoff filter for $12 \mathrm{~h}$ (see Experimental Section). ${ }^{b}$ Based on donor. ${ }^{c}$ Determined by quantitative GC using an internal standard. ${ }^{d}$ Based on reacted donor. ${ }^{e}$ These crystals did not react on photolysis at $25^{\circ} \mathrm{C}$ for $48 \mathrm{~h}$; FP and DB recovered in $>95 \%$ yield.

Importantly, the crystalline EDA complex of phenyl(pentamethylphenyl)acetylene was completely unaffected by irradiation at $\lambda_{\text {exc }}>530 \mathrm{~nm}$, even for prolonged periods of $>72 \mathrm{~h}$ at room temperature. 


\section{Time-Resolved Spectroscopic Studies of the Direct and Charge-Transfer Photoactivation of}

Crystalline EDA Complexes of Diarylacetylene and Dichlorobenzoquinone. To gain mechanistic insight into the photoinduced coupling, the crystalline EDA complexes of several prototypical diarylacetylenes (DA) in Chart 1 with dichlorobenzoquinone were exposed to 25-ps laser pulses at 355 and $532 \mathrm{~nm}$ (corresponding to the third and second harmonic, respectively, of the $\mathrm{Nd}^{3+}$ :YAG laser). The resulting transient absorption spectra were recorded on the $\mathrm{ps} / \mathrm{ns}$ time scale in the diffuse reflectance mode, $\underline{22}$ as follows.

A. $355 \mathrm{~nm}$ Excitation. Red crystals of the EDA complex of phenyl(pentamethyl) acetylene FP and quinone DB were initially irradiated at $355 \mathrm{~nm}$ as a (1 wt \%) dispersion in silica gel. Figure 4A shows the instantaneous formation of a transient spectrum consisting of an absorption band with $\lambda_{\max }=500 \mathrm{~nm}$ and a shoulder at $470 \mathrm{~nm}$. Since this absorption band was identical with one obtained from a sample of pure DB in $5 \%$ silica gel (see inset, Figure 4A), it was readily assigned to the triplet state of quinone ( ${ }^{3} \mathbf{D B}^{*}$ ) by comparison with analogous spectra of other triplet-excited quinones in solution ${ }^{23}$ and in the solid state. ${ }^{24}$ The absorption band of the triplet quinone rapidly decayed over a period of $4 \mathrm{~ns}$ to about $50 \%$ of its initial intensity (i.e., $\tau_{1 / 2} \simeq 4 \mathrm{~ns}$ ). The resulting transient absorption spectrum at $4 \mathrm{~ns}$ exhibited a new absorption band centered at $\lambda_{\max }=450 \mathrm{~nm}$ and a broad absorption extending beyond $800 \mathrm{~nm}$ (into the near-IR region). The $450 \mathrm{~nm}$ absorption was readily assigned to the quinone anion radical $\mathrm{DB}^{\circ-}$ by spectral comparison with an authentic spectrum (Figure 4B). $\underline{25}$ The broad NIR band extending beyond $800 \mathrm{~nm}$ was similarly assigned to the acetylene cation radical FP $^{\bullet+}$, which was independently generated (Figure 4C). $\underline{26 a}$

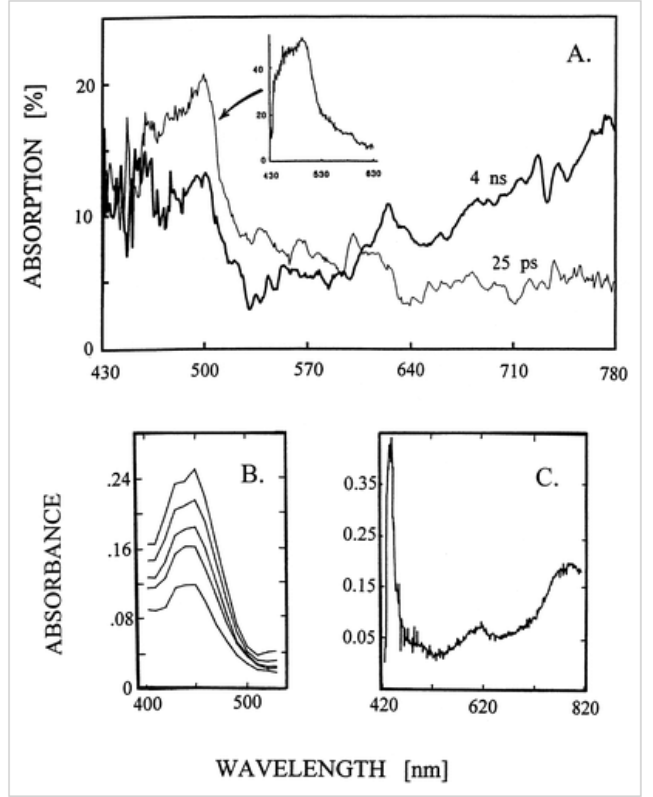

Figure 4 (A) Transient absorption spectrum obtained at 25-ps (thin line) and 4-ns (thick line) following the application of the 25-ps laser pulse at $355 \mathrm{~nm}$ to the [FP,2DB] complex in the solid state (as a 5 wt \% dispersion in silica). Inset: Transient absorption spectrum of triplet DB* obtained from the 25-ps laser pulse at $355 \mathrm{~nm}$ of dichlorobenzoquinone dispersed in silica. For comparison, the authentic 
spectrum of $(B)$ the anion radical $\mathbf{D B}^{\bullet-}$ and $(C)$ the cation radical $\mathbf{F P}^{\bullet+}$ were obtained as described in the text.

Similar time-resolved absorption spectra of diarylacetylene cation radicals $\left(\mathbf{D A}^{\bullet+}\right)$ and the quinone anion radical (DB ${ }^{*}$ ) were obtained upon the $355 \mathrm{~nm}$ laser excitation of the EDA crystals of dichlorobenzoquinone with di- $m$-xylylacetylene $\mathbf{X X}$ and $m$-xylyl(hemimellityl)acetylene $\mathbf{X H}$. Thus, in all cases, laser excitation at $355 \mathrm{~nm}$ first led to the quinone triplet $\left({ }^{3} \mathbf{D B}^{*}\right)$, which then decayed on the (ps/ns) time scale to simultaneously form the quinone anion radical (DB ${ }^{\bullet}$ ) and the diarylacetylene cation radicals $\mathbf{X X}^{\bullet+}$ and $\mathbf{H X} \mathbf{X}^{\bullet+}$, respectively.

B. 532-nm Excitation. The orange EDA crystals of phenyl(pentamethylphenyl)acetylene FP and quinone DB dispersed in silica gel (5 wt \%) were alternatively irradiated with a 532-nm laser pulse. The instantaneous spectrum in Figure 5A (observed within the 25-ps laser pulse) closely resembled the transient spectrum obtained $4 \mathrm{~ns}$ after the 355-nm excitation of the same EDA crystals in Figure 4A. Accordingly, we assigned this new absorption to the acetylene cation radial FP ${ }^{\bullet+}$ (vide supra). ${ }^{26}$ The absorption decayed to about $40 \%$ of its initial intensity within 100 ps, and the first-order decay to a raised base line (with a rate constant of $k_{\text {decay }}=2.6 \times 10^{10} \mathrm{~s}^{-1}$ ) is shown in Figure $5 \mathrm{~B} . \underline{27}$ Similar transient spectra were obtained upon the $532 \mathrm{~nm}$ laser excitation of the crystalline DB complex with di- $m$ xylylacetylene $\mathbf{X X}$. The broad absorption $(\lambda>600 \mathrm{~nm})$ decayed to $\sim 46 \%$ of its initial intensity, with a first-order rate constant of $2.3 \times 10^{10} \mathrm{~s}^{-1}$.

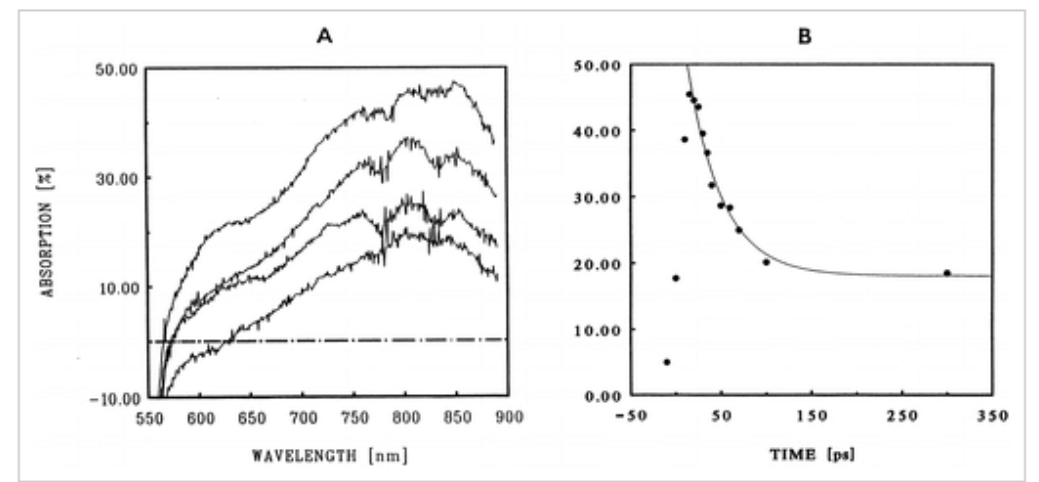

Figure 5 (A) Transient absorption spectrum obtained at (top-to-bottom) 25, 45, 70, and 100 ps following the application of the 25-ps laser pulse at $532 \mathrm{~nm}$ to the [FP,2DB] complex in the solid state. (B) Temporal decay of the spectral absorption in (A) followed at $\lambda_{\text {mon }}=808 \mathrm{~nm}$.

\section{X-ray Crystallography of Crystalline EDA Complexes of Diarylacetylenes with}

Dichlorobenzoquinone. The molecular structures of three representative examples of the crystalline EDA complexes of diarylacetylenes and 2,6-dichlorobenzoquinone were examined by X-ray crystallography. ${ }^{20}$ These encompassed a pair of photoreactive systems based on $\mathbf{T X}$ and $\mathbf{X X}$, and the unreactive complex of FP - with a particular emphasis on their structural similarities and dissimilarities, as follows. 
A. The Donor-Acceptor Interaction. In all three structures, alternating stacks of donor acetylenes and quinone are found in which there is face-to-face packing of the two quinone molecules with each of the aryl groups on the acetylene (see Figure 6). Rather surprisingly, the specific orientation of the quinone with respect to the long axis of the acetylene donor is similar in all three structures. Thus, the pairs of quinones are arranged such that each quinone is positioned above the donor with the chlorine atoms directed toward the end of the donor molecule. Furthermore, the angle between the two quinone $\mathrm{O}-\mathrm{O}$ axes and the long axis of the acetylene donor was similar: $36.8^{\circ}$ and $37.6^{\circ}$ for TX; $35^{\circ}$ for $\mathbf{X X}$, and $52.9^{\circ}$ and $41.3^{\circ}$ for FP. The interplanar distances between the planes defined by the aromatic moiety and the quinones are also similar: 3.36 and $3.39 \AA$ for TX; 3.38 and $3.39 \AA$ for XX; and 3.42 and $3.34 \AA$ for FP; and the distance between the respective reactive centers (i.e., the triple bond of the donor and the carbonyl groups of the two quinones) are as follows: $3.65 \AA$ (dimethyl aromatic ring) and $4.15 \AA$ (methylphenyl ring) for TX; $3.63 \AA$ for XX; and $4.34 \AA$ (pentamethylphenyl ring) and $3.84 \AA$ (phenyl ring) for FP.

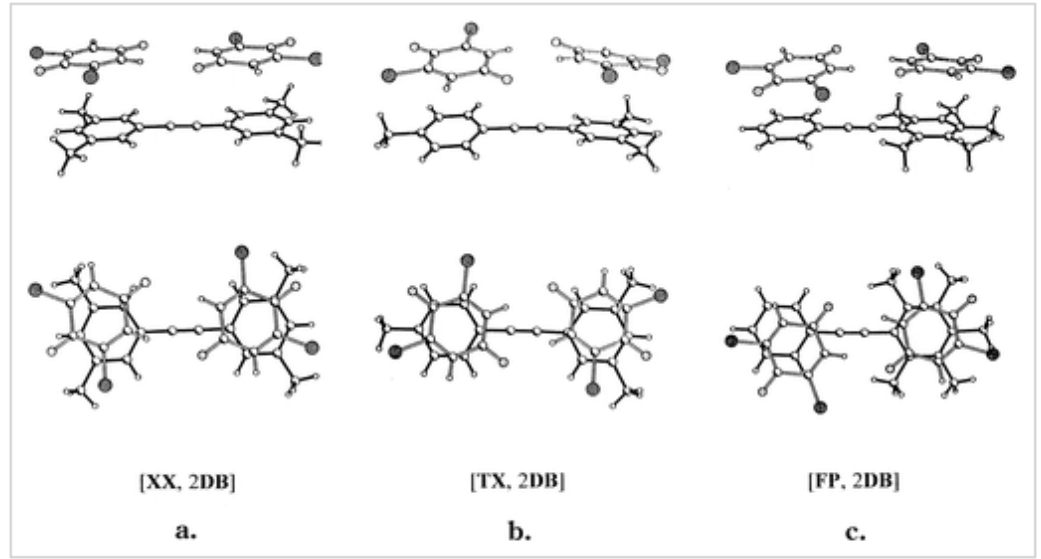

Figure 6 Two perspective views (side and top) of the crystalline 1:2 EDA complexes of diarylacetylenes and dichlorobenzoquinone, showing the disposition of the quinone DB relative to each of the polymethylated phenyl groups in the photoreactive [TX,2DB] and [XX,2DB] complexes relative to the unreactive [FP,2DB] complex in the solid state.

B. Lattice Structure. The relationship between the stacks of donors and acceptors and their neighbors vary among the three structures. Despite this, there are significant $\mathrm{Cl}-\mathrm{Cl}$ and $\mathrm{Cl}-\mathrm{O}$ interactions between the stacks. Interestingly, only the structure of [XX,2DB] shows the formation of sheets of DB that are held together by these $\mathrm{Cl}-\mathrm{Cl}$ and $\mathrm{Cl}-\mathrm{O}$ interactions. In this structure, the two aryl rings in each donor are essentially coplanar. Although the pair of quinones complexed to a particular donor do not exhibit any mutual interaction, there are $\mathrm{Cl}-\mathrm{Cl}(3.38 \AA)$ and $\mathrm{Cl}-\mathrm{O}(3.17 \AA)$ contacts to $\mathrm{Cl}$ and $\mathrm{O}$ atoms in neighboring stacks [note that the sum of the respective van der Waals radii are 3.60 and $3.2 \AA$, respectively].

In contrast, the stacks of donors and acceptors in the structures containing TX and FP are staggered with respect to each other, and planar sheets of donors or acceptors are not formed. In addition, the aryl groups of both acetylenes TX and FP are slightly twisted with respect to each other. 


\section{Discussion}

The diarylacetylenes (DA) in Chart 1 uniformly afford crystalline (1:2) donor-acceptor complexes with 2,6-dichlorobenzoquinone (DB), i.e.

\section{$\mathbf{D A}+2 \mathbf{D B} \rightleftharpoons[\mathbf{D A}, 2 \mathbf{D B}]_{\mathrm{xtl}}$}

which are characterized by distinctive colors diagnostic of charge-transfer absorptions. ${ }^{15}$ Such colored EDA complexes can be photoactivated in at least two ways - by direct irradiation of the DB acceptor or by specific CT excitation of the EDA complex via their distinctive absorption bands $\left(\lambda_{\mathrm{DB}}\right.$ and $\left.\lambda_{\mathrm{CT}}\right)$ that are illustrated in Figure 1 and tabulated in Table 1, columns 3 and 4, respectively. ${ }^{16}$

The common nature of the quinonemethide products described in Tables 2 and 3 indicates that either photochemical procedure represents a viable mode of photoactivation ( $v$ ia $\lambda_{D B}$ or $\lambda_{C T}$ ) for the coupling of diarylacetylenes with quinone. The mechanistic pathways by which such photoinduced couplings occur are clearly illuminated by the time-resolved spectral changes described in Figures 4 and 5 . In particular, the results in Figure 5 show that the specific CT excitation of the EDA complex with the 532$\mathrm{nm}$ laser pulse leads directly to the ion-radical pair state within the time scale of the 25-ps laser pulse, $\underline{28}$ i.e., Scheme 1.

$$
[\mathbf{D A}, \mathbf{D B}]_{\mathrm{xtl}} \underset{(<25 \mathrm{ps})}{\stackrel{\lambda_{\mathrm{CT}}=532 \mathrm{~nm}}{\longrightarrow}}\left[\mathbf{D A}^{+\cdot}, \mathbf{D B}^{-*}\right]_{\mathrm{xtl}}
$$

Scheme 1

By comparison, only the DB (acceptor) moiety absorbs the 355-nm irradiation in Figure 4 to instantaneously form the quinone triplet ( $\left.{ }^{3} \mathbf{D B}^{*}\right)$ within the 25 -ps laser pulse. $\frac{29}{}$ Such a photoexcited quinone is subsequently quenched by electron transfer from a neighboring diarylacetylene donor that leads to the ion-radical pair on the early nanosecond time scale, ${ }^{23}$ i.e., Scheme 2.

$$
\begin{aligned}
& {[\mathbf{D A}, \mathbf{D B}]_{\mathrm{xtl}} \underset{(<25 \mathrm{ps})}{\stackrel{\lambda_{\mathrm{DB}}=355 \mathrm{~nm}}{\longrightarrow}}\left[\mathbf{D A},{ }^{3} \mathbf{D B}^{*}\right]_{\mathrm{xtl}}} \\
& {\left[\mathbf{D A}^{3} \mathbf{D B}^{*}\right]_{\mathrm{xtl}} \underset{(\mathrm{ps} / \mathrm{ns})}{\stackrel{\text { dark }}{\longrightarrow}}\left[\mathbf{D A}^{+}, \mathbf{D B}^{-\cdot}\right]_{\mathrm{xtl}}}
\end{aligned}
$$

Scheme 2

In other words, the ion-radical pair from the acetylene/quinone complex is a common intermediate in both the direct $\left(\lambda_{\mathrm{DB}}\right)$ and the charge-transfer $\left(\lambda_{\mathrm{CT}}\right)$ photoactivation process. As such, it readily accounts for the common distribution of products in Tables 2 and 3. Furthermore, the same duality of 
photoactivation processes was found earlier to be pertinent in solution, ${ }^{17}$ and the rather free diffusion allowed in the liquid phase led to the identification of three competing processes emanating from the ion-radical pair, as summarized in Scheme 3.

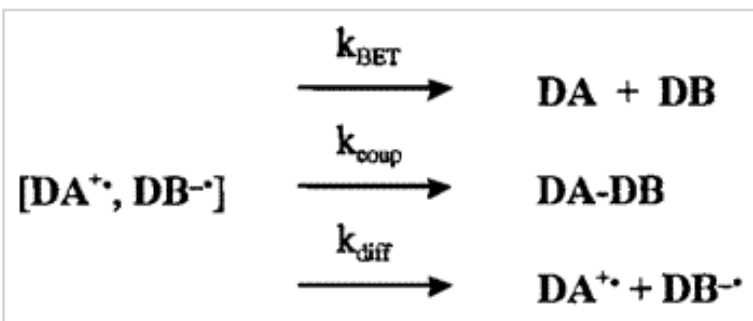

\section{Scheme 3}

Of these microdynamical processes, only the diffusive separation ( $k_{\text {diff }}$ ) is not applicable to the solid state. As such, the spectral decay in Figure $5 B$ is due to a combination of back-electron transfer ( $k_{\text {BET }}$ ) and coupling $\left(k_{\text {coup }}\right.$ ) to the distonic biradical adduct DA-DB. ${ }^{30}$ Previous studies indicate that the distonic adduct then goes on to the [2 +2$]$ cycloadduct and the quinone methide according to Scheme $4 .{ }^{17,18}$

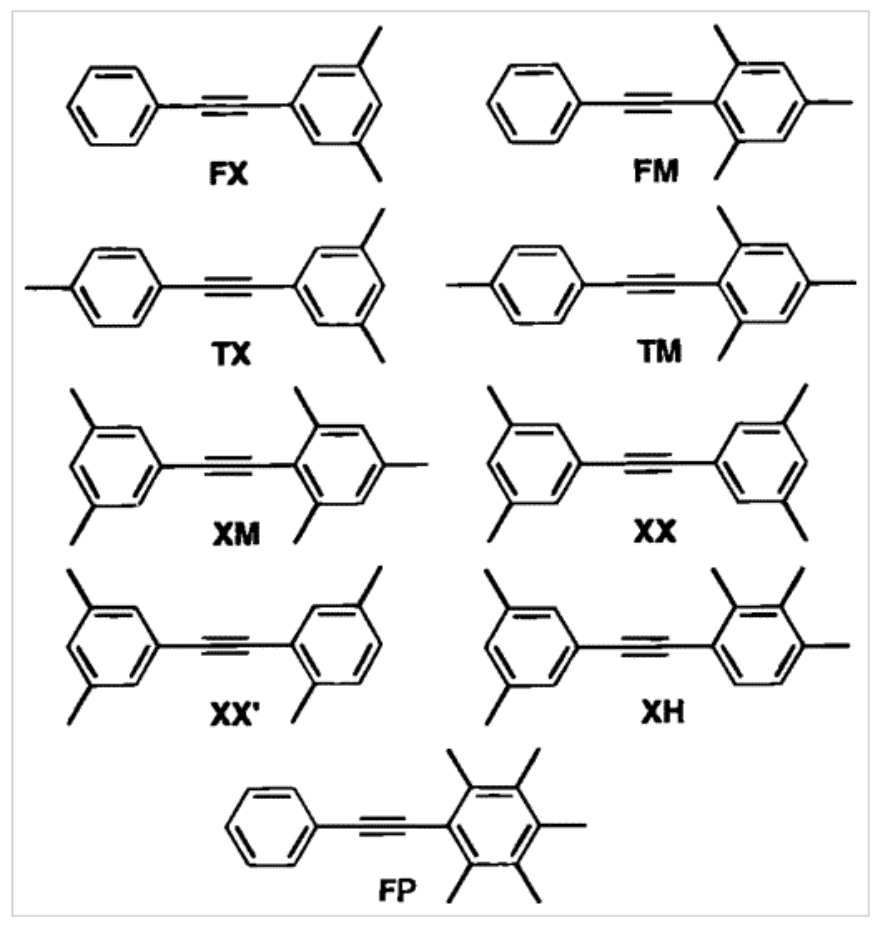

Scheme 4

Although many of the diarylacetylenes in Chart 1 afford isomeric mixtures of quinone methides that are the same in the solid state and in solution, a closer comparison of the solution/solid-state data reveals a pair of discrepancies that merit further consideration. For example, the photocoupling of tolyl- $m$-xylylacetylene (TX) and DB is significantly more selective (i.e., 1a:1b $=4: 1$ ) in the solid state 
than in dichloromethane solution (3:2). More strikingly, phenyl(pentamethylphenyl)acetylene FP is readily converted to its quinone methide with very high selectivity $(6 a: 6 b=25: 1)$ in dichloromethane solution, but the same photocoupling cannot be induced (via either $\lambda_{\mathrm{DB}}$ or $\lambda_{\mathrm{CT}}$ ) in the solid state (last entries in Tables 2 and 3).

Since the ion-radical pair is the critical intermediate in the photocouplings by either the direct or the charge-transfer excitation in solution as well as in the solid state, the different behavior must reside in how the collapse of the ion-radical pair is affected by donor-acceptor separation within the rigid crystalline lattice. For example, when the photoreactive crystals [XX,DB] and [TX,DB] are compared with the unreactive crystal, [FP,DB], the following crystallographic differences are noted in the two quinone carbonyls relative to the acetylenic group depicted in the donor-acceptor orientations below:

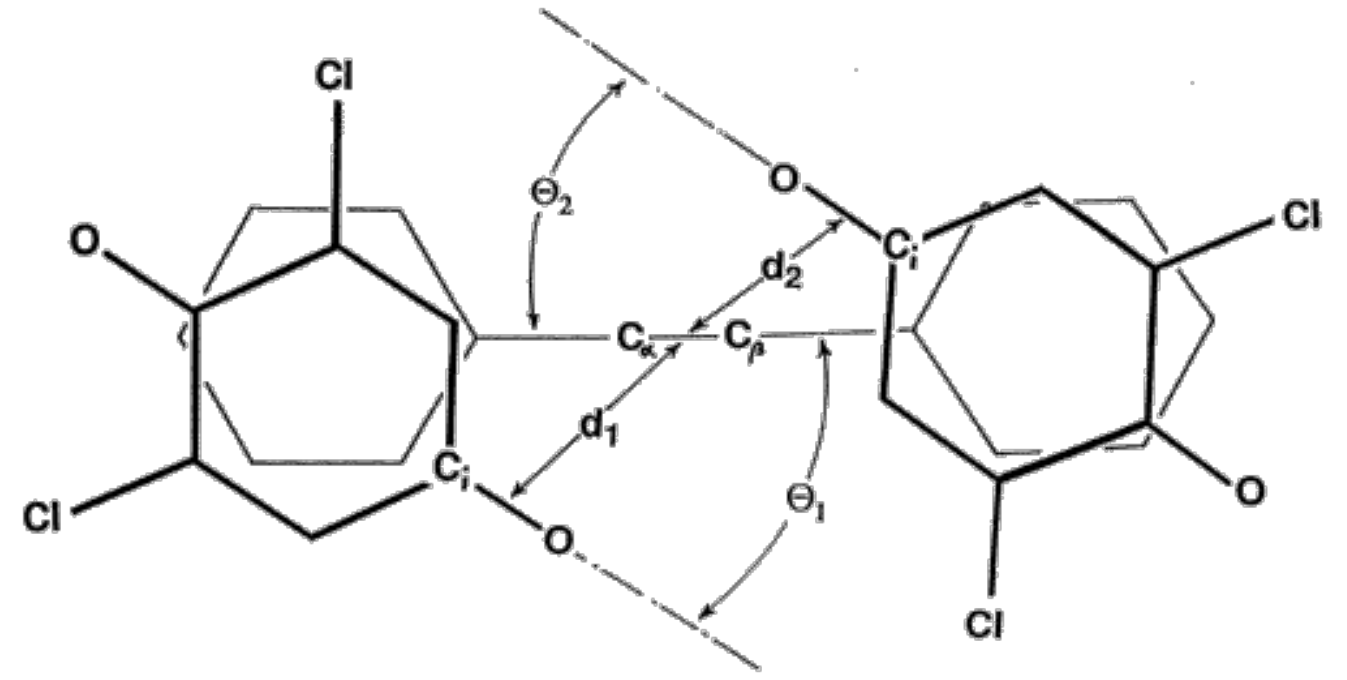

(a) In [FP,DB] the carbonyl group lies further away from the acetylene function than in either [TX,DB] or $[\mathbf{X X}, \mathbf{D B}]$, as given by the center-to-center distances $d_{1}$ and $d_{2}$ in Chart 2.

$\begin{array}{lrrrrrr}\text { Donor } & & d_{1}(\AA) & \theta_{1} \text { (deg.) } & & d_{2}(\AA) & \theta_{2} \text { (deg.) } \\ & & (\mathbf{T}) 4.15 & 36.8 & & (\mathbf{X}) 3.63 & 37.6 \\ \mathbf{X X} & & 3.63 & 35.1 & & 3.63 & 35.1 \\ \mathbf{F P} & \text { (P) } 4.34 & 41.3 & & \text { (F) } 3.84 & 52.9\end{array}$

\section{Chart 2}

(b) In [FP,DB], the overlap angles for the carbonyl/acetylene lineup are enlarged relative to those in either [TX,DB] or [XX,DB], as given by the values of $\theta_{1}$ and $\theta_{2}$ in Chart 2.

If such limited distance/angle differences are responsible for the nonreactivity of [FP,DB] in the solid state, the least-motion postulate $e^{2,31}$ suggests that [2 +2] cycloaddition arising from the collapse of the 
ion-radical pair in Scheme 3 proceeds by either $(a)$ synchronous $\left(C_{\alpha}-C_{i}\right)$ and $\left(C_{\beta}-O\right)$ bond formation directly to the oxetene intermediate or $(b)$ stepwise process involving an initial $\left(C_{\beta}-O\right)$ bond followed by a rapid ring closure of the distonic biradical adduct FP-DB to form the $\left(C_{\alpha}-C_{i}\right)$ bond. Indeed, such a simple picture would account for the common distribution of isomeric quinone methides that is by and large obtained in solution and the solid. For example, the location of the 2,6-dichloro substituents away from the acetylenic center as shown in the top perspectives (Figures 6 ) predicts the formation of only one regioisomer for DB addition to yield only the quinone methides in Tables 2 and 3.

Furthermore, the regioselectivity in the addition to the acetylenic bond always favors attack at $\mathrm{C}_{\beta}$, the less hindered center. As such, the initial charge-transfer excitation always favors the more heavily methylated phenyl group to accord with its better donor properties. $\underline{32}$

There are some facets of the least-motion postulate, however, that taken alone are not easily accommodated. For example, the very high regioselectivity in the photocoupling of [TX,DB] in the solid state to preferentially afford quinone methide $1 \mathbf{a}$ is difficult to rationalize. Thus, the quinone that must react to afford $1 \mathrm{a}$ is that positioned above the $m$-xylyl group, but further away from the acetylenic linkage $\left(d_{1}=4.15 \AA\right.$ vs $d_{2}=3.63 \AA$ ), although clearly still within the bounds defined by Schmidt. ${ }^{2}$ [Note the overlap angles $\theta_{1}$ and $\theta_{2}$ are comparable]. If such differences of $d_{1}$ and $d_{2}$ are not a factor, then why is [FP,DB] so unreactive? Part of the explanation may lie in the large steric volume occupied by the large pentamethylphenyl group to significantly alter the reaction cavity as defined by Cohen. $\underline{33}$ Another factor that we cannot evaluate at this juncture is the possibility that the stepwise route to the oxetene via the distonic biradical (Scheme 4) may actually allow time for a pendant aryl group to flip around ${ }^{31 b}$ (and thus violate the least-motion postulate). Further studies are clearly required to delineate such subtleties in the solid-state photocoupling involved with EDA complexes. $\frac{34}{4}$

\section{Summary and Conclusions}

The [2 + 2] cycloaddition of diarylacetylene (DA) to dichlorobenzoquinone (DB) can be achieved in the solid (crystalline) state by photoinduced electron transfer. The diarylacetylene donors in Chart 1 form crystalline (1:2) EDA complexes with the quinone, and X-ray crystallography establishes the cofacial dispositions in Figure 6 that allow either (a) the charge-transfer absorption band at $\lambda_{C T}>530 \mathrm{~nm}$ or (b) the quinone absorption at $\lambda_{D B}>410 \mathrm{~nm}$ to be specifically irradiated. In both cases, photocoupling leads to the quinone methides in Tables 2 and 3 to be obtained in high yields and regioselectivities. Timeresolved (ps/ns) diffuse-reflectance spectroscopy reveals the ion-radical pair $\left[\mathbf{D A}^{\bullet+}, \mathbf{D B}^{{ }^{*-}}\right.$ ] as the first reactive intermediate, which then undergoes coupling via the ion-pair collapse to the distonic adduct DA-BA, as previously described for the same photocouplings carried out in dichloromethane solution. ${ }^{17}$ Indeed, the close comparison of the photocoupling products in the solid state and in solution indicate some striking similarities, but the detailed analysis based on the least motion postulate requires further evaluation.

\section{Experimental Section}

Materials. The methyl-substituted diphenylacetylenes were prepared in the previous study ${ }^{17}$ by the palladium-catalyzed coupling of the corresponding aryl iodides and arylacetylenes in diethylamine. 2,6- 
Dichlorobenzoquinone (Aldrich) was used as received. Dichloromethane (Mallinckrodt, reagent grade) was purified by standard procedures. $\underline{35}$ UV-vis absorption spectra were recorded on a Varian Cary- 5 UV-vis spectrometer equipped with a Varian DRA-2041393 diffuse-reflectance accessory. Gas chromatography was performed on a Hewlett-Packard 5890A series gas chromatograph equipped with a HP 3392 integrator. GC-MS analyses were carried out on a Hewlett-Packard 5890 chromatograph interfaced to an HP 5970 mass spectrometer (EI, $70 \mathrm{eV}) .{ }^{1} \mathrm{H}$ and ${ }^{13} \mathrm{C}$ NMR spectra were recorded in $\mathrm{CDCl}_{3}$ on a General Electric QE-300 NMR spectrometer, and the chemical shifts are reported in ppm units downfield from tetramethylsilane. Melting points were performed on a Mel-Temp (Laboratory Devices) apparatus and are uncorrected. Elemental analysis was performed by Atlantic Microlab Inc., Norcross, GA.

Preparation of Crystalline EDA Complexes of Diarylacetylene Donors with 2,6-Dichlorobenzoquinone. When a mixture of the colorless crystalline donor TX (44 mg, $0.2 \mathrm{mmol}$ ) and canary yellow crystals of DB (72 $\mathrm{mg}, 0.4 \mathrm{mmol}$ ) was prepared in a test tube, the solid mixture took on a bright orange coloration. Dichloromethane $(2 \mathrm{~mL})$ was added and the test tube wrapped in aluminum foil and placed in the refrigerator at $-20^{\circ} \mathrm{C}$. The dichloromethane evaporated over the period of 5 days, and orange needles slowly grew. Quantitative GC and elemental analysis confirmed the 2:1 mixture of DB and TX. Anal. Calcd for $\mathrm{C}_{29} \mathrm{H}_{20} \mathrm{Cl}_{4} \mathrm{O}_{4}: \mathrm{C}, 60.65 ; \mathrm{H}, 3.51$. Found: $\mathrm{C}, 60.91 ; \mathrm{H}, 3.68$. In experiments performed with an excess of either the quinone (i.e., a 1:4 ratio of TX:DB) or an excess of donor (i.e., a 2:1 ratio of TX:DB), the same (2:1) orange crystals were obtained together with the excess of either uncomplexed component. It is noteworthy that evaporation of the dichloromethane at $20^{\circ} \mathrm{C}$ resulted in the deposition of microcrystalline solids that were unsuitable for X-ray crystallography. Moreover, a similar procedure that employed a variety of solvents, diethyl ether, acetonitrile, hexane, pentane, tetrahydrofuran, chloroform, and mixtures of these solvents, did not yield suitable crystals.

Colored crystals were similarly grown from dichloromethane solutions of the quinone DB with the diarylacetylenes $\mathbf{X X}, \mathbf{T M}, \mathbf{X M}, \mathbf{X H}, \mathbf{X X}$, and FP. The analyses were as follows. [XX,2DB]. Anal. Calcd for $\mathrm{C}_{30} \mathrm{H}_{22} \mathrm{Cl}_{4} \mathrm{O}_{4}:$ C, 61.25; $\mathrm{H}, 3.77$. Found: $\mathrm{C}, 61.12 ; \mathrm{H}, 3.80$. [XX',2DB]. Anal. Calcd for $\mathrm{C}_{30} \mathrm{H}_{22} \mathrm{Cl}_{4} \mathrm{O}_{4}: \mathrm{C}_{2}, 61.25$; $\mathrm{H}$, 3.77. Found: $\mathrm{C}, 61.19 ; \mathrm{H}, 3.87$. [XM,2DB]. Anal. Calcd for $\mathrm{C}_{31} \mathrm{H}_{24} \mathrm{Cl}_{4} \mathrm{O}_{4}: \mathrm{C}, 61.82 \mathrm{H}, 4.02$. Found: $\mathrm{C}$, 61.64; $\mathrm{H}, 4.08$. [XH,2DB]. Anal. Calcd for $\mathrm{C}_{31} \mathrm{H}_{24} \mathrm{Cl}_{4} \mathrm{O}_{4}: \mathrm{C}, 61.82 \mathrm{H}, 4.02$. Found: $\mathrm{C}, 61.94 ; \mathrm{H}, 4.07$.

[FP,2DB]. Anal. Calcd for $\mathrm{C}_{31} \mathrm{H}_{24} \mathrm{Cl}_{4} \mathrm{O}_{4}: \mathrm{C}, 61.82 \mathrm{H}, 4.02$. Found: $\mathrm{C}, 61.53 ; \mathrm{H}, 4.14$. Solid mixtures of FM with DB (2 equiv) melted together to form a red oil, and we were unable to grow suitable crystals for $\mathrm{X}$ ray crystallography.

Spectral (UV-Vis) Characterization of the Crystalline EDA Complexes. The 1:2 crystalline solids from the graded series of donors in Chart 1 and DB were characterized by a progressive reddening of the solid, which was correlated to the extent of methyl substitution of the donor. Thus, while mixtures of DB with TX yielded pale orange crystals, $\mathbf{X X}$ yielded orange crystals, and dark red crystals were formed from FP. To quantify these effects we recorded the UV-vis diffuse-reflectance spectra of these crystals. For example, a solid mixture of the orange crystalline complex [TX,2DB] in silica (4 mg in $400 \mathrm{mg}$, i.e., 1 wt \%) was ground until a homogeneous mixture was obtained and the solid transferred into a 1-mm quartz cuvette. The diffuse reflectance spectrum of the pale orange solid was recorded [the base line 
was based on a well-ground sample of silica gel in the same cuvette]. Next, the diffuse reflectance spectrum of DB was recorded as a mixture in silica, and the concentration of DB matched the concentration of DB used to record the spectrum of the colored complex (2.5 mg in $400 \mathrm{mg}$ ). The intensity of the local absorption band of DB at $350 \mathrm{~nm}$ was approximately the same in both spectra, and the digital subtraction of the latter spectrum from the former generated a broad absorption spectrum with a maximum at $500 \mathrm{~nm}$ (Figure 1B). Similar treatment of the other crystalline EDA complexes yielded the absorption maxima listed in Table 1.

Photoinitiated Solid-State Coupling of Diarylacetylenes with 2,6-Dichlorobenzoquinone. A. Direct Excitation. To a thin Pyrex tube were added several orange crystals ( $7 \mathrm{mg}, 0.012 \mathrm{mmol}$ ) of the EDA complex [XX,2DB], and the tube was sealed under an argon atmosphere. The reaction tube was placed in a clear Dewar filled with acetone cooled to $-60^{\circ} \mathrm{C}$ and the sample irradiated with a mediumpressure mercury lamp fitted with an aqueous IR filter and a Corning cutoff filter to effect irradiation with visible light $\left(\lambda_{\text {exc }}>410 \mathrm{~nm}\right)$. The tube was rotated each hour, and the temperature in the Dewar was controlled by the periodic addition of dry ice. After $5 \mathrm{~h}$ of irradiation, the crystals examined under a microscope were found to be darker and extensively cracked. The crystals were dissolved in dichloromethane, and the immediate analysis by quantitative GC indicated a $15 \%$ conversion of the donor acetylene to form a single product. The crude products from several experiments were combined and purified by thin-layer chromatography with hexane/ethyl acetate (10/1) as the eluant. The structure of the quinonemethide product was established by NMR and GC-MS to be identical with that previously formed in solutions. ${ }^{17}$ It should be noted that irradiation of the EDA crystals was performed at $-60^{\circ} \mathrm{C}$, since the irradiation of [TX,2DB] at $20^{\circ} \mathrm{C}$ resulted in the total loss of crystallinity (to form a melt). The other acetylene donors in Table 2 were similarly irradiated at $-60^{\circ} \mathrm{C}$, with the various conversions and yields listed. The structures of the products were established by direct comparison of the GC, GC-MS, and ${ }^{1} \mathrm{H}$ NMR spectra with the products of the corresponding photocouplings previously carried out in dichloromethane solution. ${ }^{17}$

B. Charge-Transfer Excitation. The orange (2:1) crystals of TX with DB ( $9 \mathrm{mg}, 0.015 \mathrm{mmol}$ ) were similarly sealed (under an argon atmosphere) in a thin Pyrex tube and placed in a clear Dewar filled with acetone cooled to $-60^{\circ} \mathrm{C}$. The crystals were irradiated with a medium-pressure $\mathrm{Hg}$ lamp (500 W) filtered through an aqueous IR filter and a Corning cutoff filter $\left(\lambda_{\text {exc }}>530 \mathrm{~nm}\right)$. After $12 \mathrm{~h}$, the solid was dissolved in dichloromethane and analyzed by quantitative GC. The products were identified by comparative GC-MS with the quinone methides already identified in the solution phase reaction. ${ }^{17}$ The conversions and yields are listed in Table 3.

Time-Resolved Diffuse-Reflectance Spectroscopy. For the time-resolved diffuse-reflectance spectroscopy, the crystals containing the 2:1 EDA complexes of DB with diarylacetylenes were ground to a fine powder, mixed with silica gel (60-200 mesh) to form $5 \mathrm{wt} \%$ dispersions, and stored in 1-mm quartz cuvettes. The laser apparatus for the picosecond diffuse-reflectance spectroscopy has been previously described in detail. $\underline{36}$ Briefly, the second $(532 \mathrm{~nm})$ or the third $(355 \mathrm{~nm})$ harmonic output of a mode-locked Nd:YAG laser (QUANTEL, YG 501-C, 25-ps fwhm) was used as the excitation source. The (residual) fundamental (1064 nm) laser beam was focused onto a 10-cm cuvette containing a 50:50 
mixture of $\mathrm{H}_{2} \mathrm{O}$ and $\mathrm{D}_{2} \mathrm{O}$ to generate white supercontinuum pulses of 25 -ps duration. The white light was split into two beams that served as reference light and probe light for the powder samples. The diffuse-reflected probe light as well as the reference light were picked up by fiber optics and fed into a flat-field spectrograph to which a dual-diode-array detector (Princeton Instruments) was attached. The spectra were represented as percentage absorption (\% ABS) as defined by $\% \mathrm{ABS}=100\left(1-R / R_{0}\right)$, with $R$ and $R_{0}$ representing the intensities of the diffuse-reflected probe light and of the reference light, respectively.

\section{Acknowledgment}

We thank the R. A. Welch Foundation and the National Science Foundation for financial support.

\section{References}

${ }^{1}$ For a comprehensive review of organic solid-state photochemistry: Ramamurthy, V.; Venkatesan, $\mathrm{K}$. Chem. Rev. (Washington, D.C.) 1987, 87, 433.

${ }^{2}$ For a review see: Schmidt, G. M. J. Pure Appl. Chem 1971, 27, 647.

${ }^{3}$ Cohen, M. D.; Schmidt G. M. J.; Sonntag, F. I. J. Chem. Soc. 1964, 2000.

${ }^{4}$ See also: (a) Fu, T. Y.; Liu, Z.; Scheffer, J. R.; Trotter, J. J. Am. Chem. Soc. 1993, 115, 12202. (b) Enkelmann, V.; Wegner, G.; Novak K.; Wagener, K. B. J. Am. Chem. Soc. 1993, 115, 10390.

${ }^{5}$ This distance was determined empirically from studies of the photodimerization of cinnamic acid derivatives. $^{2,3}$ Subsequently, several photoinduced dimerizations have been reported where the distance between reactive olefins is $\geq 4.45 \AA .^{6}$

${ }^{6}$ See: Gnanaguru, K.; Ramasubbu, N.; Venkatesan, K.; Ramamurthy, V. J. Photochem. 1984, $27,355$.

${ }^{7}$ (a) Kaup, G.; Jostkleigrewe, E.; Herman, H. J. Angew. Chem., Int. Ed. Engl. 1982, 21, 435. (b) Hasegawa, M.; Kato, S.; Saigo, K.; Wilson, S. R.; Stern, C. L.; Paul, I. C. Photochem. Photobiol. A 1988, 41, 385. (c) Nalini, V.; Desiraju, G. R. Tetrahedron 1987. 43, 1313.

${ }^{8}$ For reviews, see ref 2 and: Kaupp, G. In Organic Photochemistry and Photobiology; Horspool, W. M., Song, P. S., Eds.; CRC Press: New York, 1994.

${ }^{9}$ See: Gnanaguru, K.; Ramasubbu, N.; Venkatesan, K. Ramamurthy, V. J. Org. Chem. 1985, 50, 2337 and previous papers in the series.

10 The cocrystallization of different cinnamamides was reported by Schmidt: Leiserowitz, L.; Schmidt, G. M. J. J. Chem. Soc. A 1969, 2372.

${ }^{11}$ Sarma, J. A. R. P.; Desiraju, G. R. J. Am. Chem. Soc. 1986, 108, 2791.

12 For an early review, see: Prout, C. K.; Kamenar, B. In Molecular Complexes; Foster, R., Ed.; Elek Science: London, 1973; Chapter 4.

${ }^{13}$ Rathore, R.; Lindeman, S. V.; Kochi, J. K. J. Am. Chem. Soc. 1997, 119, 9393.

${ }^{14}$ Foster, R.; Foreman, M. I. In The Chemistry of the Quinonoid Compounds; Patai, S., Ed.; Wiley: New York, 1974; Part 1, Chapter 6.

${ }^{15}$ Foster, R. Organic Charge-Transfer Complexes; Academic: New York, 1969.

${ }^{16}$ Kochi, J. K. Acta Chem. Scand. 1990, 44, 409.

17 Bosch, E.; Hubig, S. M.; Kochi, J. K. J. Am. Chem. Soc., in press.

${ }^{18}$ For the original report, see: (a) Zimmerman, H. E.; Craft, L. Tetrahedron Lett 1964, 2131. (b) BryceSmith, D.; Fray, G. I.; Gilbert, A. Tetrahedron Lett. 1964, 2137. (c) Creed, D. In CRC Handbook of 
Organic Photochemistry and Photobiology; Horspool, W. M., Song, P.-S., Eds.; CRC Press: Boca Raton, FL, 1995. The oxetene in eq 3 is short-lived but can exhibit lifetimes of up to several hours depending on the temperature. See: (d) Friedrich, L. E.; Bower, J. D. J. Am. Chem. Soc. 1973, 95, 6869. (e) Friedrich, L. E.; Lam, P. Y.-S. J. Org. Chem. 1982, 46, 306. (f) Friedrich, L. E.; Schuster, G. B. J. Am. Chem. Soc. 1971, 93, 4602.

19 (a) Mulliken, R. S. J. Am. Chem. Soc. 1950, 72, 600. (b) Mulliken, R. S. J. Am. Chem. Soc. 1952, 74, 811. (c) Mulliken, R. S. J. Phys. Chem. 1952, 56, 801. (d) Mulliken, R. S.; Person, W. M. Molecular Complexes; Wiley: New York, 1969.

${ }^{20}$ Deposit with the Cambridge Crystallographic Data Centre, 12 Union Road, Cambridge, CB2 1EZ, U.K.

${ }^{21}$ The favored MS fragmentation of benzoyl groups is described: McLafferty, F. W.; Turecek, F. Interpretation of Mass Spectra, 4th ed.; University Science: Mill Valley, CA, 1993.

22 Wilkinson, F. J. Chem. Soc., Faraday Trans 2 1986, 82, 2073. See also: Hubig, S. M.; Kochi, J. K. J. Phys. Chem. 1995, 99, 17578.

${ }^{23}$ (a) Gschwind, R.; Haselbach, E. Helv. Chim. Acta 1979, 62, 941. (b) Hubig, S. M.; Bockman, T. M.; Kochi, J. K. J. Am. Chem. Soc. 1997, 119, 2926. (c) Johnston, L. J.; Schepp, N. P. J. Am. Chem. Soc. 1993, 115, 6564. (d) Levin, P. P.; Kuz'min, V. A. Russ. Chem. Rev. 1987, 56, 307. (e) Kobashi, H.; Funabashi, M.-A.; Kondo, T.; Morita, T.; Okada, T.; Mataga, N. Bull. Chem. Soc. Jpn. 1984, 57, 3557.

${ }^{24}$ Hubig, S. M. Unpublished results.

25 (a) The authentic spectrum of DB •- was obtained by electron-transfer quenching of the photoexcited (triplet) DB* with a dibenzofuran donor in acetonitrile. Note the cation radical of the donor does not absorb in the $400-500 \mathrm{~nm}$ region. (b) The narrow absorption band of the cation radical $\mathbf{F P}^{\bullet+}$ at $430 \mathrm{~nm}$ could not be observed owing to the low intensities of the diffusereflected probe light at this wavelength.

${ }^{26}$ (a) The authentic spectrum of FP'+ ${ }^{\bullet}$ was obtained by charge-transfer excitation of the EDA complex of FP with maleic anhydride in dichloromethane. Note the anion radical of maleic anhydride does not absorb in the detection window between 400 and $800 \mathrm{~nm}$. (b) The strongly scattered excitation light at $532 \mathrm{~nm}$ precluded any transient absorption measurements below $550 \mathrm{~nm}$ in the diffuse-reflectance mode.

27 Such a kinetics behavior was previously observed upon the photoexcitation of other crystalline EDA complexes. For a discussion of the phenomenon, see Hubig et al. in ref 22.

${ }^{28}$ Direct photoactivation of the EDA complex produces the singlet ion pair in eq 10 (Scheme 1). See: (a) Ojima, S.; Miyasaka, H.; Mataga, N. J. Phys. Chem. 1990, 94, 4147. (b) Wynne, K.; Galli, C.; Hochstrasser, R. M. J. Chem. Phys. 1994, 100, 4797.

${ }^{29}$ Recent time-resolved (fs) spectroscopy established the initially formed singlet excited quinone to undergo ultrafast intersystem crossing to the triplet state with $k_{\text {isc }}=10^{11} \mathrm{~s}^{-1}$ : Hubig, S. M.; Bockman, T. M.; Kochi, J. K. J. Am. Chem. Soc. 1997, 119, 2926.

${ }^{30}$ From the solution studies, ${ }^{17}$ back-electron transfer $\left(k_{\mathrm{BET}}\right)$ is roughly 10 times faster than coupling ( $\left.k_{\text {coup }}\right)$.

${ }^{31}$ (a) The least-motion postulate predicts $\left(C_{i}-C_{\alpha}\right)$ and $\left(C_{\beta}-O\right)$ bond formation to be more or less synchronous. (b) Such a flip involves a sequential $\left(C_{\alpha}-O\right)$ and $\left(C_{i}-C_{\beta}\right)$ bond formation.

${ }^{32}$ Howell, J. O.; Goncalves, J. M.; Amatore, C.; Klasinc, L.; Wightman, R. M.; Kochi, J. K. J. Am. Chem. Soc. 1984, 106, 3968.

${ }^{33}$ Cohen, M. D. Angew. Chem., Int. Ed. Engl. 1975, 14, 386. 
34 The related Paterno-Büchi coupling of stilbenes and quinone will be reported later.

${ }^{35}$ Perrin, D. D.; Armarego, W. L. F.; Perrin, D. R. Purification of Laboratory Chemicals; Pergamon: New York, 1980.

${ }^{36}$ Yoon, K. B.; Hubig, S. M.; Kochi, J. K. J. Phys. Chem. 1994, 98, 3865. 